XIII.

Aus der pathologisch-anatomischen Abteilung des Karolinischen medikochirurgischen Instituts in Stockholm (Vorstand: Prof. Dr. Carl Sundberg).

\title{
Ein Fall von zentral in der Niere gelegenem Hypernephrom.
}

\author{
Von \\ Nils Stenströnt. \\ (Mit 2 Abbildungen im Text.)
}

Seitdem Grawitz1) im Jahre 1883 seine Erfahrungen über einige Nierengeschwülste, von ihm Strumae lipomatodes aberrantae renis, später nach Birch-Hirschfeld öfter Hypernephrome genannt oder vielleicht am öftesten als Grawitz'sche Geschwülste bezeichnet, veröffentlichte, ist unzähligemal die Abstammung dieser Geschwülste abgehandelt. Das Verdienst Grawitz' liegt besonders darin, daß er die Eigenart der Geschwïlste hervorhebt und gezeigt hat, daß sie nicht, wie zu seiner Zeit angenommen wurde, Lipome waren. Infolge der auffälligen Aehnlichkeit des Geschwulstgewebes mit Nebennierenrinde und mit der Kenntnis, daß Teile des Nebennierenparenchyms als aberrante Keime ebenfalls unter der Nierenkapsel sich bisweilen finden, lag es sehr nahe, wie Grawitz ${ }^{2}$ ), anzunehmen, daß diese Geschwiilste von solchem Gewebe ausgegangen waren. Im allgemeinen war diese Annahme der Nebennierenabstammung der Hypernephrome ohne Widerspruch angenommen. Der erste, der eine ernsthafte Kritik daran wagte, war Sudeck ${ }^{3}$ ), der rein morphologischen Untersuchungen zufolge die fraglichen Geschwïlste als vom Nierengewebe ausgegangene Adenome erklärte. Diese Kühnheit rief die Vertreter der Grawitz'schen Lehre, vor

1) Grawitz, P., Die sogen. Jipome der Niere. Virchow's Arch. Bd. 93. 1883. S. 39.

2) Grawitz, P., Die Entstehung von Nierentumoren aus Nebennierengewebe. Arch. 1. klin. Chir. 1884. Bd. 30. S. 624.

3) Sudeck, P., Ueber die Struktur der Nierenadenome. Thre Stellung zu den Strumae suprarenales abberatae (Grawitz). Virchow's Arch. 1893. Bd. 133. S. 405 . 
allem Lubarschi), zu einer hitzigen Polemik gegen Sudeck auf und die Angaben Sudeck's wurden als grundlos erklärt. In den letzten Jahren ist wieder die Lehre der Nebennierenabstammung angegriffen worden vor allem in Arbeiten von Zehbe ${ }^{2}$ ) und Stoerk ${ }^{3}$ ) - Zehbe gibt einen ausführlicheren Bericht des Streites über die Abstammung der Grawitz'schen Geschwülste.

Die Methoden, die, um die Abstammung der fraglichen Geschwülste ins Reine zu bringen, angewandt wurden, sind teils chemische, teils ververgleichend anatomische und teils morphologische.

Chemisch ist teils das Glykogen und teils die Fettsubstanzen Gegenstand der Untersuchung gewesen, und solche Untersuchungen, unter anderen von Sisson ${ }^{4}$ ) und Rost ${ }^{5}$ ) ausgefiihrt, haben gezeigt, daß es hierin große Aehnlichkeiten, aber auch wesentliche Verschiedenheiten zwischen Nebennieren und Grawitz schen Geschwülsten gibt. Eine endgültige Lösung der Frage haben diese Untersuchungen nicht gegeben, und tnter anderen warnt Rost davor, weitumfassende Schlüsse aus der chemischen Zusammensetzung des Gewebes zu ziehen.

Steink $\mathrm{e}^{6}$ ) hat durch vergleichende Untersuchungen tierischer Geschwülste das Problem zu lösen versucht und deshalb mit Material von Rindern und Pferden gearbëitet. Bei diesen Tieren scheinen indessen nicht Nierengeschwülste, sondern nur Nebennierengeschwülste, den Grawitzschen Geschwïlsten beim Menschen einigermaßen ähnlich, vorzukommen.

Die Methode, die die wertvollsten Aufklärungen gegeben hat, ist jedoch die rein morphologische. Abgesprengte Keime von Nebennierenparenchym.kommen, wie Untersuchungen von Marehand ${ }^{7}$ ), Chiari ${ }^{8}$ ),

1) Lubarsch, O., Beiträge zur Histologie der von Nebennierenkeimen ausgehenden Nierengeschwülste. Virchow's-Arch. 1894. Bd. 135. S. 149. - Lubarsch; 0., Ueber die Abstammung gewisser Nierengeschwülste von embryonal abgesprengten Nebennierenkeimen. Virchow's Archiv. 1894. Bd. 137. S. 191.

2) Zehbe, M., Untersuchungen über Nierengeschwïlste. Virchow's Arch. Bd. 201. S. 150 .

3) Stoerk, 0., Zur Histogenese der Grawitz schen Nierengeschwülste. Ziegler's Beitr. 1908. Bd. 43. S. 393.

4) Sisson, W. R., Zur Frage der Grawitz schen 'Tumoren. Ziegler's Beitr. 1910. Bd. 49. S. 476.

5) Rost, Fr., Zur Differentialdiagnose von primären Ḱnochenendotheliomen und Hypernephrommetastasen nebst Beitrag zur Histogenese der Grawitz'sohen Tumoren. Virohow's Arch. 1912. Bd. 208, 1912. S. 53.

6) Stein ke, P., Untersucbungen zur vergleichenden Pathologie der Nebennierengeschwülste. Frankfurter Zeitschr. f. Pathol. 1910. Bd. 5.

7) Marchand, Ueber akzessorisehe Nebennieren im Ligamentum latum. Virchow's Arch. Bd. 92. S. 11.

8) Chiari, Zeitschr. f. Heilkunde. Bd. 5. 
Schmorli) und andere gezeigt haben, im Plexus solaris und dessen Umgebung vor, wie auch unter der Leber- und der Nierenkapsel und längs des Verlaufes der Vena spermatica nach den Ovarien bezw. den Hoden. Als Stätze seiner Theorie der Abstammung der fraglichen Geschwülste von solchen aberranten Nebennierenteilen führt Grawitz unter anderem die Lage der Geschwülste ganz unter der Nierenkapsel oder zwischen zwei Renkuli an, welcher Platz auch der unvergleichbar gewöhnlichste Fundort aberranter Nebemieren ist. Grawitz und seine Nachfolger haben jedoch übersehen, was zuerșt Stoerk deutlich hervorhebt, nämlich das eigentümliche darin, daß aberrante Nebennieren nirgends als in der Niere Geschwulstwachstum verursachen, obgleich sie, wenn auch viel seltener, auch in anderen parenchymatösen Organen wie der Leber, wo, wie es dünkt, die Möglichkeiten zu weiterer Entwickelung gleichartig wie in der Niere sein würden, vorkommen. Schon diese Tatsache muß ein kräftiges Argument sein beim Verleugnen der Nebennierenabstammung der Grawitzschen Geschwïlste, besonders weil Forscher, die die aberranten Nebennieren studiert haben, wie z. B. Marchand, die grobe Geneigtheit solchen abgesprengten Parenchymes in kurzer Zeit zu versehwinden, deutlich hervorheben, indem aberrante Nebennieren beinahe ausschließlich bei Neugeborenen gefunden werden. Warum würde dann solches Parenchym in einem Organ mit so großen eliminativen und resorptiven Möglichkeiten wie die Niere fortleben? Auch das selbe Aussehen der Geschwülste hat Anlab zu Zweifeln betreffs ihrer Nebennierenabstammung gegeben und vor allem hat hier die Lumenbildung eine Rolle gehabt, da Lumina, die oft in den Grawitz'schen Geschwïlsten gefunden werden, nimmer in den Nebennieren oder aus denselben ausgegangenen Geschwülsten angetroffen werden.

Nach einem eingehenden Studium eines großen Materials kommen auch - wie einmal vorher Sudeck - unabhängig von einander beinahe gleichzeitig Zehbe und Stoerk zu der Schlußfolgerung, daß die Grawitzschen Geschwülste rom Nierengewebe ausgegangen sein müssen und nicht von einigen in die Niere eingelagerten fremden Organelementen, speziell Nebemierenteilen. Welche Nierenteile es sind, aus denen diese speziellen Gesehwülste hervorwachsen, kann noch nicht gesagt werden. Daß man mit abgesprengten Keimen $z u$ rechnen hat, darin dünkt man einig zu sein, aber ob diese schon bei der Geburt als abgesprengt vorkommen oder es erst später werden, kann nicht mit Sicherheit entschieden werden. Untersuchungen von embryonalen Nieren, die unter anderen Busse $^{2}$ ) gemacht hat, zeigen indessen oft Bilder, die eine gewisse Aehr-

1) Schmorl, G., Zur Kenntnis der akzessorischen Nebennieren. Ziegler"s Beiträge. 1891. Bd. 9. S. 523.

2) Busse, 0., Ueber. Bau, Entwickelung und Etnteilung der Nierengeschwälste. Virchow's Arch. 1899. Bd. 157. S. 346 a. 377. 
lichkeit mit entwickelten Nierenadenosarkomen zeigen, und Sisson (l.c.) lenkt die Aufmerksamkeit auf die beiden Möglichkeiten der Entwickelung aus metanephrogenen Keimen und aus solchen von dem Harnleiterteil der Niere abstammend. Andererseits sieht man selten oder niemals Hypernephrome schon beim Neugeborenen sich entwickeln, sondern sie entstehen erst bei solchem Alter, wo das Individuum sehr wohl Infektionen durchgemacht haben kann, die Nierenalterationen mit nachfolgenden Verschrumpfungen und Absprengungen von Parenchymteilchen bedingt haben können. Grawitz'sche Geschwülste sind, wie es aus der Literatur hervorgeht, speziell gewöhnlich in Schrumpfnieren, und wenn auch Geschwulstwachstum aus entzündlich oder traumatisch abgesprengtem Parenchym ungewöhnlich ist, dürfte doch diese Möglichkeit nicht ganz verneint werden können. Die entzündlichen Prozesse können ja auch, wie mehrseits hervorgehoben wird, schon längst vorher abgesprengte Keime zu Proliferation anreizen.

Kongenital dürfte solch eine Absprengung am leichtesten in den Zusammenschmelzungsstellen der verschiedenen Renculi zustande gebracht werden, wie auch dort die größten Gelegenheiten zur Einschließung von Nebennierenkeimen bestehen. Der Fundort von aus Teilen des Nierenparenchyms entstandenen Geschwülsten sollte aber auch gelegentlich tiefer in der Niere vorkommen. Grawitz'sche Geschwülste von solcher'Lage sind indessen selten. Zehbe beschreibt zwei solche tief in der Niere beim Nierenbecken gelegene Hypernephrome. Wenn auch solche Fälle an sich - ohne Rücksicht auf die morphologischen und chemischen Verschiedenheiten der Geschwülste und des Nebennierengewebes, die die genannten Verfasser ausfïhrlich beschreiben - nicht als ein absoluter Beweis gegen die Lehre der Nebennierenabstammung angesehen werden können, so sprechen sie doch sehr dagegen, indem aberrante Nebennierenkeime subkapsulär oder höchstens etwa $1 \mathrm{~cm}$ in die Nierensubstanz hineinragend gelegen sind. Abgesehen von diesen beiden Fällen Zehbe's sind von mehreren Verfassern mehr zentral gelegene Hypernephrome beschrieben worden, aber es handelt sich hier um große Geschwülste, die bei ihrem Wachstum aus einem oberflächlichen Entstehungszentrum bis in die Tiefe des Nierenbeckens gereicht haben. Da, woraul schon hingewiesen ist, zentral gelegene Hypernephrome als eine Stütze der Lehre der nephrogenen Abstammung angesehen werden können, dürfte es von Interesse sein, daß solehe, wenn sie angetroffen werden, zur allgemeinen Kenntnis kommen.

Ich habe zur Untersuchung ein Präparat, durch Operation einer 46jährigen Frau gewonnen, bekommen, das eine solche ungewöhnliche Lage eines Hypernephroms darstellt. Das fragliche Präparat besteht aus einer linken Niere, $13,5 \mathrm{~cm}$ lang, $9,5 \mathrm{~cm}$ breit und $6 \mathrm{~cm}$ dick. Die zentralen Teile dieser Niere sind von einem mandaringroßen, mehrknotigen, 
264 Stenström, Ein Fall ron zentral in der Niero gelegenem Hypernophrom.

mittels einer dïnnen bindegewebigen Kapsel gut abgegrenzten Geschwulst eingenommen, die makroskopisch wie mikroskopisch den für Hypernephrome charakteristischen Typus hat. Der zentrale Teil der Geschwuist ist im Nierenhilus gelegen, die Peripherie desselben erreicht an der Vorderseite der Niere die Nierenkapsel in einem kinderhandgroßen Gebiet und zeichnet sich dort als eine grobknotige Tumormasse ab. Der konvexe Nierenrand, die beiden Nierenpole und die hintere Fläche der Niere be-

Abbildung 1.

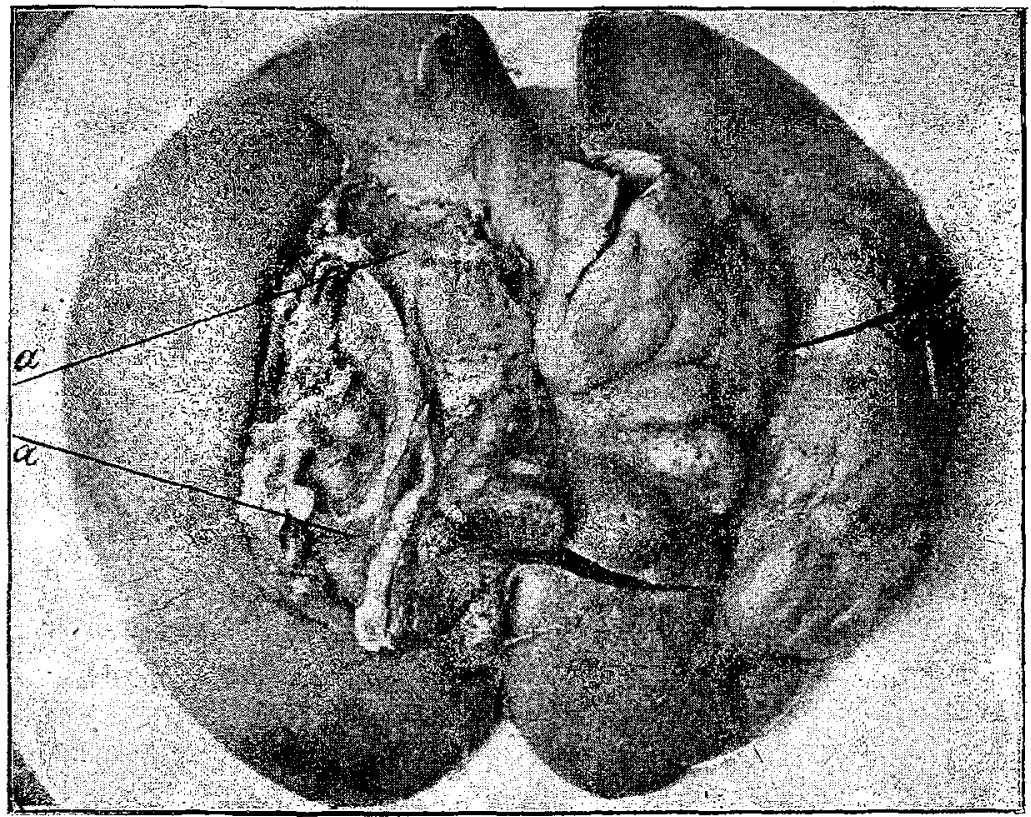

$a=$ Nierenkelche.

stehen im großen und ganzen aus Nierenparenchym verschiedener Dicke. Dieses Nierengewebe besteht hanptsächlich aus Rinde, die, wo sie an die Geschwulst grenzt, Veränderumgen mit verdrängten und sklerosierten Glomeruli und mit fachem Endothel ansgekleideten kieinen Zysten zeigt, und schlieblich beim Tumorgewebe in ein fibröses Kapselgewebe îbergeht.

Die Harnwege dieser Niere haben einen ziemlich komplizierten Verlauf. Der Harnleiter besteht, wie aus Abb. 1 hervorgeht, aus 2 Nierenkelchen, die, dem Umkreis der Geschwulst folgend, sich später in ein System dïnner spaltförmiger Räume an der Grenze zwischen Nicre und 
Geschwulstgewebe verzweigen, in welchen Spalten die intrarenalen Harukanälchen einmünden.

Im Zentrum der eigentlichen Geschwulst am Sitze des Nierenbeckens einer normalen Niere gibt es ein zentrales System unregelmäßiger

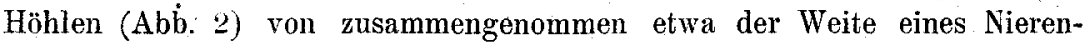
beckens. Von diesem zentralen Teil gehen Ausläufer durch das Geschwulstparenchym, ein System von gegen die Peripherie immer schmäler werdenden

\section{Abbildung 2.}

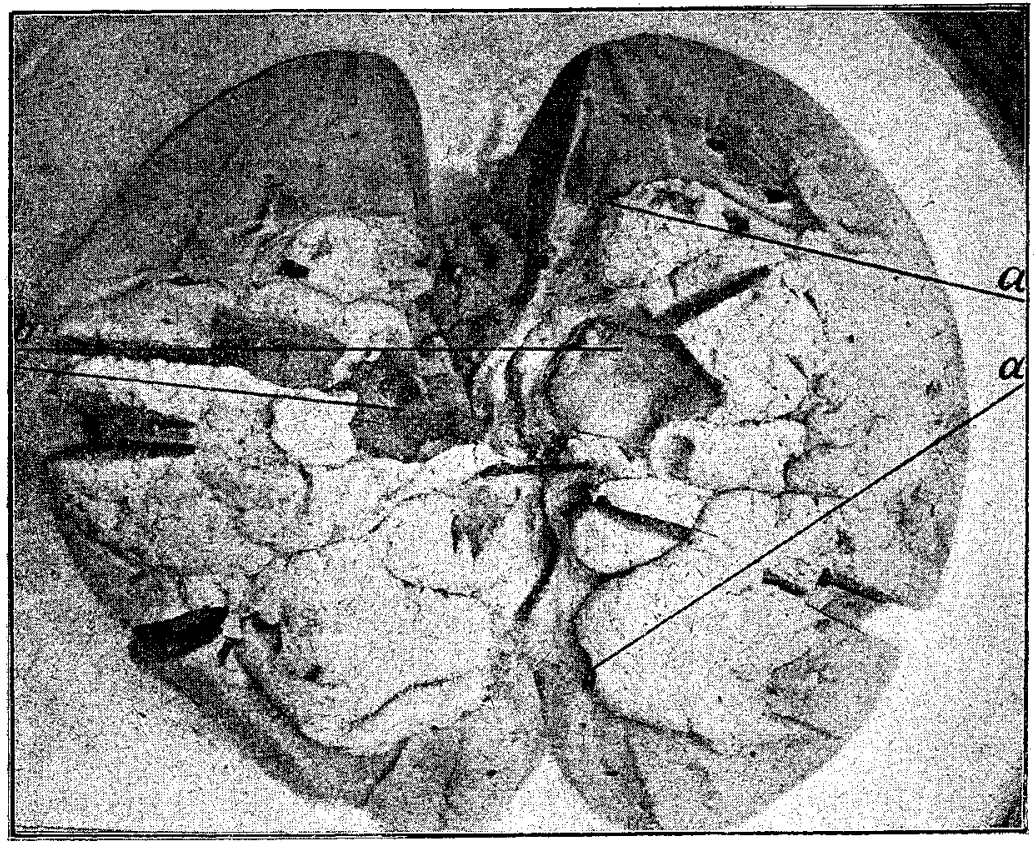

$\mathrm{a}=$ Nierenkelche. $\mathrm{b}=$ Zysten der Geschwulst.

Röhren, hier und da zu etwas weiteren Höhlen dilatiert, und schließlich in spaltförmigen Kavitäten an der Grenze zwischen Geschwulst- und Nierengewebe einmündend. Diese Höhlen der Geschwulst sind wie die kleinen Zysten der Geschwulstkapsel und des angrenzenden Nierengewebes von einem niedrigen Endothel auf einer Bindegewebsmembran, die gegen die zentralen Teile mit ihren größeren Höhlen immer dicker wird, bekleidet.

Höhlenräume dieser Art, wie die hier vorkommenden, sind als in gewissem Maß charakteristisch für Hypernephrome beschrieben, und das Vorkommen derselben ist unter anderem als etwas der Nebennierentheorie 
Widersprechendes angeführt worden. Die Deutung der Höhlenräume als Resorptionszysten nach Blutungen ist in dem fraglichen Falle wegen ihres Baues und des Aussehens der. Ungebung ausgeschlossen. Es bleibt dann sur übrig; sie als Rekentionszysten Sekretes irgendeiner Ari zu deuten; man könnte dam an Produkte von Geschwulstgewebe, einschließlich der Blutund Lymphbahnen, denken oder auch an Harn. Da man im Mikroskope sieht, wie die Hohlräume im Umkreis der Geschwulst an zahlreichen. Stellen in sub- und intrakapsuläre Räume, die unzweideuqig dorch Sekretretention entstanden sind, übergehen, dürfte-indessen die Anmahme an nächsten liegen, dạ sie als Retentionszysten des Harnes zu deuten sind. Fine generalisierte solche Deutung erklärt, warum ähnliche Hohlräume oder Zysten nicht in den Nebennierenadenomen vorkommen. Die Zysten wiirden spezifische Bildmgen der in der Niere vorkommenden Geschwilste fraglicher Art sein ind in Zusammenhang mit den vergrößerten Schwierigkeiten der Elimination des Nierensekretes entstehen; wenn ähnliche Geschwülste in anderen Organen vorkamen, würden sie sehr wohl der Zysten ermangeln, ohne daß diese Verschiedenheit als eine wesentlich andersartige Wuchsart des Geschwulstgewebes zu denten wäre. Die angefullute leutung setzt natürlicherweise nicht voraus, dab die Geschwiniste vom Nierengewebe ausgegangen sind, wenn auch Harnretentionszysten in einer Nierengeschwulst leichter und natürlicher entstehen sollen, als in einer Geschwulst fremder Elemente. Wenn also mit einer Erklärung, wie der angetühton, einer der emsteren. Einwände gegen die Nebennierentheorie älterer Zeiten wegfällt, haben doch spätere oben angedentete Ergebnisse der Forschung die-nephrogene Entstehung wenn auch nicht sichergestellt. so doch wahrsicheinlicher gemacht.

Das Geschwulstgewebe im übrigen bietet kein spezielles Interesse. Es: wird zum größten' Teil von stark verfetteten, in Präparaten, wo das Fett durch die Präparierungsart weggenommen worden ist, stark vakuolisierten Zellen mit netzförmigem Protoplasma und kleinem rundlichen Kern, in. Schläuche geordnet mit einem dünnen gefäBführenden Bindegewebsstroma, gebildet. An anderen Stellen sind die Zellen 'kleiner mit mehr honagenem Protoplasma und an manchen Orten tubulär mit Andentung von Lumina geordnet. Rücksichtlich des Gehalts an Glykogen habe ich wegen der Konservierung des Materiales keine Untersuchungen ausführen können. Hier und $d a$ in der. Geschwulst finden sich Blutungen von im ganzen ziemlich unbeträchtlicluer Ausdehnung. Die beschriebenen Hohlräume erscheinen in einzelnen zwecks mikroskopiseher Untersuchung ausgeschnittenen Stücken in der Weise, daß sie sehr wohl als kleine geschlossene Zysten in der Geschwalst gedeutet werden könnten, wenn nicht ihr gegenseitiger Zusammenhang schon erkannt war, und die Möglichkeit, 
daß ähnliche Hohlräume von anderen Verfassern als geschlossene Zysten gedeutet sind, liegt sehr nahe.

Betreffs der zentralen Entstehung der Geschwulst kann dagegen eingewandt werden, daß eine solche nicht für sicher bewiesen angesehen werden kann, da das Geschwulstgewebe in einem bedeutenden Bereich die Nierenkapsel erreicht. Es ist indessen unwahrscheinlich, daß eine anfangs oberflächlich gelegene Geschwulst ihren ursprünglichen Zelltypus beibehaltend und ohne mehr hervortretende Zeichen von Malignität, bei ihrem Wachstum eine so zentrale Lage einnehmen würde, wenigstens spricht eine solche Auffassung gegen alles, was bisher von dem Wachstum der Hypernephrome bekannt ist. Der Ausgangspunkt der Geschwulst muß als zentral in der Nähe des Nierenhilus oder bei demselben gelegene Keime gedacht werden, welche später bei ihrem Wachstum nicht nur sämtliche Nierenpyramiden, sondern anch die Rinde der vorderen Nierenfläche verdrängt haben. Das letztgenannte würde dafür sprechen, daB die ursprünglichen Keime mehr nach dieser Seite gelagert gewesen sind. Eine solche tiefe Lage stimmt nicht mit dem, was von der Lage abgesprengter Nebennierenkeime bekannt ist. Da die Hypernephrome sämtlich, wenn sie so klein sind, daß eine solche Bestimmung gemacht werden kann, in der Rindensubstanz der Niere gefunden werden, ist es wahrscheinlich, daß diese, in einiger Weise modifiziert, die Matrix der Geschwülste sind, und dak die Entstehungskeime der beschriebenen Geschwulst in dem zentralen Teil einer Columna Bertini der ${ }_{3}$ yorderen Hälfte der Niere zu suchen ist.

\section{Resümee.}

Beschreibung eines mandarinengroßen, histologisch benignen Hypernephroms im Nierenhilus gelegen, sämtliche Nierenpyramiden verdrängend, teilweise die Nierenoberfläche erreichend:

Ein System ron in der Geschwulst gelegenen Hohlräumen, in der Peripherie mit intrakapsulären Höhlen von gleichem Bau wie die Zysten des teilweise schrumpfenden Nierengewebes in Zusammenhang stehend, wird als Harnretentionszysten gedeutet.

Die Identität dieser Höhlen mit den als charakteristisch für die Hypernephrome von den Autoren beschriebenen "Lumina“. wird in Frage gestellt. 